\title{
Acción Inmediata hacia una Paternidad más Responsable *
}

\author{
Dr. Gonzalo Echeverry Parra**
}

\section{a) LA PLANIFICACION FAMILIAR CON- VENCIONAL DEJA A LOS "POBRES" POR FUERA DE SUS PROGRAMAS.}

En la década de 1960/69 tuvo lugar la aparición y florecimiento de las campañas de Planificación Familiar. La preocupación por los problemas concernientes al acelerado crecimiento de la población, que ya venía manifestándose con caracteres cada vez más apremiantes desde los años 50 , y el advenimiento de los anticonceptivos modernos (píldora y DIU), crearon las condiciones necesarias para que se establecieran en el tercer mundo los primeros programas de planificación familiar.

Dificultades emanadas de circunstancias culturales, políticas o religiosas, además del no muy claro conocimiento de los efectos secundarios inmediatos y tardíos de los anticonceptivos en uso, hicieron que estos programas incipientes se rodearan de todas las precauciones posibles para no acrecentar las resistencias existentes y poder sobrevivir y prosperar. De aquí surgió un modelo de Clínica de Planificación Familiar por el cual comenzaron todos los programas gubernamentales o privados, con riguroso examen de las candidatas a anticoncepción por calificados especialistas en Ginecología, toma de extendidos vaginales para detección precoz de cáncer cervical, consulta de ginecología y cuidados prenatales, intervención de psicólogos y trabajadores sociales, además de estricto seguimiento médico con nuevos y prolijos exámenes practicados por especialistas. Las

\section{* Presentado en el Simposio Internacional} de Tokio Abril de 1977.

**Director Planeación y Desarrollo - Profamilia. condiciones de la época justificaban entonces un sistema que por sus elevadísimos costos y por la manifiesta escasez de personal médico apenas permitía unas pocas clínicas en los principales centros urbanos, sin poder llegar a la gran masa de la población.

Pero había tres ausentes de aquellos complicados y costosos programas clínicos: la población rural, los habitantes de los barrios marginados de las grandes ciudades, y los de los núcleos urbanos menores a donde no alcanzó el radio de acción de las pocas clínicas existentes. Dicho en otros términos, la planificación familiar convencional llegó a cubrir un pequeño grupo de habitantes urbanos y dejó por fuera a la gran mayoría de la población de los países subdesarrollados, o sea los que denominaremos con el nombre genérico de "Pobres".

"Pobres", porque no tienen la educación mínima para poder informarse de que las familias pueden planificarse y cómo hacerlo; "Pobres", porque no tienen la independencia de criterio necesaria para apartarse de influencias político-religiosas que les impiden satisfacer la necesidad sentida de regular racionalmente su fecundidad; "Pobres", porque no conocen la importancia de una paternidad responsable y consciente: "Pobres", en fin, porque no tienen el dinero para comprar los servicios profesionales o los elementos materiales que son necesarios para una planificación familiar razonable y humana.

\section{b) PORQUE SE DEJA POR FUERA A LOS "POBRES"?}

Además de las razones que constituyen su "pobreza", son múltiples las causas para que éstos hayan quedado al margen de los 
servicios clínicos convencionales de planificación familiar:

1. E1 rechazo a los exámenes pélvicos, unas veces por elemental sentido del pudor que, como sabemos, va en proporción inversa al nivel educativo, y otras veces por motivos de carácter cultural o religioso:

2. El deseo de privacidad: al acudir a una clínica de planificación familiar, la usuaria queda automáticamente identificada como alguien que está practicando la anticoncepción, cosa que en muchas ocasiones es considerada inconveniente;

3. Esa especie de autoselección que hace que las personas colocadas en los últimos peldaños de la escala socio-económica sientan una invencible timidez de acercarse a centros que consideran muy sofisticados para su miseria:

4. La inexistencia misma de los servicios: Países apenas en vía de desarrollo, con médicos y enfermeras totalmente insuficientes y mal distribuídos y con servicios rurales de salud que dejan tanto que desear o son prácticamente inexistentes, nunca podrán pensar en extender la planificación familiar a escala nacional con base sólo en programas de tipo clínico. La planificación familiar como programación horizontal dentro de los servicios gubernamentales de salud, tropieza con la debilidad de la infraestructura $\mathrm{y}$ a veces con la timidez en las decisiones, para no mencionar la crónica penuria en que dichos servicios se debaten.

5. La prioridad que los Gobiernos y Asociaciones de Planificación $\mathrm{F}$ amiliar dan a los servicios clínicos en las ciudades, muy explicable ya que se trabaja en un medio en donde cualquier esfuerzo tiene un resultado cuantificable e inmediato, a diferencia del trabajo en áreas habitadas por "Pobres".

6. Muy pocos gobiernos, Asociaciones privadas o personas llegan a darse cuenta de que los "Pobres" han quedado por fuera de los programas.

El éxito fácil del programa clínico urba- no ha polarizado su atención hacia esa actividad, y se desconoce, a veces de buena fe, que hay una inmensa mayoría de población a quien no se está prestando la menor atención y que pasa desapercibida, como si no residiera allí el verdadero problema familiar, social $y$ demográfico.

\section{c) LA PLANIFICACION FAMILIAR CO- MUNITARIA ES UNA SOLUCION SATISFACTORIA.}

El advenimiento de los años 70 trajo interesantes cambios en los conceptos de planificación familiar y en la conformación de las campañas.

La actitud general dejó de ser hostil para hacerse poco a poco más favorable; surgió lentamente la necesidad de extender la información y servicios de planificación familiar a sectores mucho más amplios de la población, y se vió cómo los servicios clínicos resultaban imprácticos y costosos que hacían imposible su expansión.

Diez años de experiencia afianzaron la confianza en los métodos anticonceptivos, al hacer un balance favorable entre los beneficios evidentes y los riesgos potenciales; el número de mujeres que utilizan la píldora anovulatoria por autoprescripción aumentó rápidamente año tras año; la experiencia de algunos países en cuanto a delegación de ciertas funciones de salud en personal no médico comenzó a ser aceptada, y la demanda de servicios de planificación familiar fué cada vez mayor.

Así empezó a conformarse el nuevo concepto de distribución no clínica de los anticonceptivos. con base en la cooperación de la comunidad, como una solución satisfactoria, aunque no perfecta, para extender la planificación familiar a los núcleos poblacionales no protegidos por los servicios clínicos. Decimos que es una solución satisfactoria, porque:

1. Crea y utiliza nuevos canales de comunicación para llevar la información a grupos urbanos marginales o a la población rural, apartándose de los sistemas convencionales y llega así más fácilmente a personas que son por naturaleza desconfiadas y tímidas, pero que tienen 
una gran capacidad de asimilación cuando se les transmiten los mensajes en lenguaje apropiado y con técnicas que les hagan sentir que la planificación de la familia es su problema y no el problema de una institución, grupo o gobierno.

2. Suprime los inconvenientes ya anotados para los servicios clínicos en cuanto no requiere un personal profesional costoso y escaso, no somete a las usuarias a exámenes que provocan rechazo, no señala o identifica a las personas que planifican sus familias, elimina las pérdidas de tiempo y dinero por recorrer largas distancias hasta encontrar los servicios clínicos, etc.

3. Por tratarse de programas de carácter centrífugo que van hacia el usuario, donde quiera que éste resida, en vez de esperar a que el usuario venga hacia los programas, multiplica casi al infinito el muy limitado alcance geográfico de las clínicas.

4. Los precios subsidiados para los anticonceptivos eliminan la barrera de los altos costos para los usuarios. No tiene sentido un programa comunitario con anticonceptivos a precios comerciales.

5. La participación de la comunidad misma en el desarrollo de las actividades de promoción y distribución es uno de los elementos más significativos del sistema. Una vez que se han involucrado elementos voluntarios que no reciben salario y que por esta misma razón merecen la confianza especial de sus comunidades, éstas toman el programa como cosa propia, lo defienden y mantienen, y le quitan todo aspecto de imposición política, institucional o de clase, para hacer de él algo propio que va en beneficio de la comunidad y que la enorgullece. Es esta la espina dorsal de la planificación familiar de base comunitaria, y su verdadera razón de ser.

6. Los costos de un programa comunitario pueden en un comienzo ser elevados cuando se lo establece en áreas rurales, por la dispersión de las comunidades; pero a medida que se organizan los sistemas de comunicación y de distribución, los costos del programa comunitario vienen a ser cada vez menores y a colocarse muy por debajo de los programas clínicos, pudiendo llegar a la larga a tener autosuficiencia económica, si se dispone de anticonceptivos donados o adecuadamente subsidiados.

7. La utilización de la píldora anovulatoria como anticonceptivo no clínico ha sido el paso más atrevido pero también el más importante en esta modalidad de programas de planificación familiar. Sin este nuevo enfoque no hubiera sido posible montar un programa comunitario eficaz, y la solución habría estado muy lejos de ser satisfactoria. Obviamente, la planificación familiar comunitaria se hará más fácil a medida que vayan apare. ciendo anticonceptivos mejores, y llegará a ser el sistema imperante cuando se disponga del anticonceptivo ideal que tanto esperamos pero que se encuentra todavía tan lejano.

d) Qué es Planificación Familiar Comunitaria?

Podríamos decir que Programas Comunitarios son aquellas acciones que crean la demanda con mecanismos apropiados de comunicación y satisfacen esa demanda con sistemas de distribución que ponen al alcance geográfico y financiero de todos, los elementos necesarios para el control razonable de la fecundidad. Dentro de este marco de referencia, se harán las adaptaciones que en cada país o región se consideren importantes para que el programa no choque contra ningún principio cultural, político, legal, religioso o tradicional.

\section{e) La Planificación Familiar Comunitaria es factible en todas partes.}

Dentro de los delineamientos generales expresados cabe toda una serie de modalidades múltiples que hacen posible el establecimiento de la Planificación Familiar Comunitaria en cualquier país, cultura o religión en donde haya que extender el alcance de los servicios clínicos de planificación familiar. Prueba de ello son los programas existentes hoy en día en distintos países, que pueden clasificarse en cuatro grupos más o menos definidos (Population Reports, J5 ,Julio 1975) :

La Distribución Institucional, que busca hacer llegar los anticonceptivos a la población rural a través de agencias o puntos de 
entrega de instituciones existentes, como ocurre en Filipinas con la Iglesia de Cristo y el Banco de San Luis o en India con el Instituto Agrícola de Allanhabad.

La Distribución Comercial de Anticonceptivos a precios subsidiados se está haciendo principalmente a base de condones que se hacen llegar al usuario por canales comerciales preexistentes tales como distribuidores de té, baterías, aceite de comer, cigarrillos, etc. S eda a los condones un nombre atractivo y se hace una propaganda comercial como para cualquiera otro producto.

Los ejemplos más representativos son los programas de India (Nirodh), Kenya (Kinga), Srilanka (Preethi) y Jamaica (Panther).

La Distribución Puerta a Puerta ha sido ensay ada principalmente en Taiwan', Korea y . Egipto con buenos resultados iniciales pero con el alto costo que representa mantener suministros permanentes por este sistema.

Los programas realmente comunitarios que se basan en activa promoción por personal de dedicación exclusiva, y en disctribución y mantenimiento da la continuidad por voluntarios pertenencientes a las comunidades, parecen ser los que han logrado mayor impacto, a juzgar por los tres ejemplos más importantes: Tailandia (CBFPS), Brasil (BEMFAM) y Colombia (PROFAMILIA).

Son todos programas privados que, a pesar de unas múltiples limitaciones, han alcanzado cualquiera de ellos en muy breve tiempo cifras de usuarias por encima de los 100.000 casos activos.

Permítaseme traer como ejemplo el programa de Profamilia, Colombia, y mostrar cómo cuando se basaba sólo en clínicas, con grandes esfuerzos humanos y financieros se logró llevar el servicio a 39 ciudades grandes o medianas, más de la mitad de ellas por encima de los 100.000 habitantes, mientras que los programas comunitarios, rurales y urbanos, están cubriendo hoy en día 352 municipalidades de las 942 en que está dividido el país desde el punto de vista político-administrativo.
Puede pensarse que los servicios gubernamentales de salud podrán alcanzar un cubrimiento no sólo igual sino superior mediante servicios clínicos prestado por los Centros de Salud; pero recordemos que aquí la planificación familiar viene a ser una actividad secundaria que se deja casi siempre para cuando no hay otra cosa más urgente y que los "Pobres" siguen teniendo muchas reservas hacia los programas clíniços: dos razones para que los resultados de programas basados exclusivamente en servicios gubernamentales de salud hayan dado un resultado modesto, al menos en América Latina. En cambio, complementados éstos con un plan de distribución comunitaria, se amplía notoriamente el radio de acción.

f) Cómo establecer un programa Comunitario de Planificación Familiar.

Para establecer programas comunitarios de planificación familiar se requieren condiciones indispensalbes sin las cuales es inútil pretender tener éxito con este nuevo enfoque.

Antes que nada, hay que identificar el problema. Hay que darse cuenta de que existen los "Pobres", aquéllos a quienes no llega la planificaicón familiar convencional por las razones atrás expuestas pero que,, sin embargo, tienen la necesidad potencial sentida de limitar razonablemente su fecundidad. Habrá que descorrer el velo de los ojos de los que toman las decisiones, ya que pueden estar artificialmente cegados por ignorancia, por información mala o tendenciosa, o por la simple pereza mental que los hace pensar que con unas cuantas actividades clínicas que alcanzan apenas un pequeño porcentaje de la población, se está cumpliendo con el deber de facilitar la planificación familiar.

El reconocimiento e identificación del problema tiene que venir necesariamente acompañado de la convicción dé que es importante y de que necesita una solución. No basta saber o aceptar que existe el problema; hay que estar intimamente convencidos de su gravedad y de la justicia social que implica su solución. 
Reconocido el problema y convencidos de su trascendencia, hay que estar decididos a resolverIo, es decir, a establecer un sistema que alcance a los "Pobres" en la mayor medida posible. Esta decisión significa estar dispuestos a enfrentar con serenidad y valor las dificultades que inevitablemente han de surgir provenientes de gobiernos, profesión médica y paramédicas, agrupaciones religiosas, entidades comerciales (droguistas), agrupaciones políticas, etc.

Un programa comunitario es algo que no se improvisa de un momento para otro, y que tropieza con enormes dificultades mientras se supera la etapa de aceptación inicial; hay que imponerlo con el valor que nace de la decisión, cuando ésta, a su vez, ha sido originada en una convicción profunda y en un generoso deseo de servir.

Ya se mencionó atrás cómo la planificación familiar de base comunitaria debe buscar y utilizar cualquier infraestructura preexistente que represente canales fáciles de comunicación con las comunidades marginadas, rurales o urbanas. Todo servicio de extensión agrícola de mejoramiento social o familiar, de salud pública de acción comunal, etc., que esté disponible, debe ser utilizado al máximo. No se puede esperar a que estas instituciones busquen o propongan la cooperación con planificación familiar: hay que buscar y proponer con insistencia dichos contactos, para no tener que crear con grandes dificultades y a muy altos costos lo que está creado, y aprovechar así la confianza y el ascendiente que otras entidades han conseguido ante los "Pobres".

Es muy importane tener en cuenta las circunstancias legales en cada programa tiene que desenvolverse. Es obvio que no se trata de estimular actividades que vay an en contra de claras disposiciones legales, pero debe tenerse en cuenta que en la mayor parte de los países del tercer mundo se encuentra una legislación más o menos permisiva que hay que aprovechar. Cuando las leyes vayan taxativamente en contra de los programas comunitarios, habrá que iniciar acciones tendientes a modificar las normas existentes para hacerlas favora- bles o, por lo menos, más alésticas.

Hay que tener un profundo respeto por las culturas y tradiciones de las comunidades sobre las que se va a actuar. La base del programa es la cooperación de la comunidad misma $y$, por consiguiente, en ningún momento se irá en contra de ella.

Las comunidades son extremadamente celosas en la guarda de sus tradiciones y de sus herencias culturales. Si lo programs son en alguna manera opuestos a ellas, se buscará cambiar los conceptos, demostrando la razón que nos acompaña y la bondad de nuestros puntos de vista.

Para que cualquier actividad de distribución tenga éxito, hay que crear la demanda.

En planificación familiar la demanda no surge por generación espontánea o por la simple disponibilidad de suministros en cantidades más o menos grandes.

El mensaje sobre planificación familiar ha de llevarse a los "Pobres" con base principalmente en los beneficios que una paternidad responsable y una familia planificada traen a la familia misma y a las personas que la componen. En otros términos, a las familias a quienes queremos llegar hay que tocarles su propio problema en vez del problema demográfico-económico ó el problema de salud pública, que no es el suyo y que no comprencerán nunca, precisamente por ser "Pobres". Aquello está bien para los gobiernos, pero no para los modestos habitantes de las áreas rurales o de los tugurios de miseria de las ciudades.

Cuando comprendamos que planificación familiar no es nada diferente de una protección eficaz contra el embarazo indeseado y que es un derecho humano protegerse contra él como se protege contra cualquiera otra enfermedad, será siempre

fácil encontrar el mensaje y el medio para llevar la información necesaria a cualquier grupo humano y bajo cualquiera circunstancia de educación, escolaridad, religión, raza, estado socio-económico, etc. 
Queda finalmente, uno de los aspectos más controvertidos, pero al mismo tiempo más indispensables de los programas comunitarios. Se quiere dar información y servicio en planificación familiar a los "Pobres" de las áreas rurales y áreas urbanas marginadas pero conservando al mismo tiempo requerimientos técnicos y académicos que son imposibles de trasplantar a programas que, para lograr algún éxito, tienen que caracterizarse por su simplicidad. Hay, pues, que renunciar al profesionalismo en todos los campos y aceptar con honestidad que hoy, lo mismo que a través de toda la historia de la humanidad, puede practicarse la anticoncepción sin intervención directa de personal profesional, que es lo que los "Pobres" necesitan y piden. Los médicos debemos deponer algunos de los conceptos tradicionales de nuestra formación, aceptar la realidad tal como es sin querer traspasarla al plano de lo teórico y de lo ideal, ser otra vez pioneros en los nuevos enfoques de la planificación familiar, comprender el alcance social de nuestra profesión y prestar así un servicio más a la comunidad humana.

Los sociólogos tendrán que aceptar que la necesidad de la planificación familiar en una comunidad "Pobre" puede palparse sin una prolija encuesta de Conocimientos, Actitudes y Prácticas, y no condicionar la existencia de los programas a investigaciones previas. Los evaluadores y estadísticos deberán conformarse con sistemas extremadamente simples de registro y monotonía de programas para que puedan ser llevados por elementos pertenecientes a una comunidad de "Pobres". Los estudios $e$ investigaciones especiales deben ser también una consecuencia de los programas y no una condición previa para ellos; en otros términos, evaluar los programas y no proyectar éstos alrededor de un complejo mecanismo evaluativo.

Los psicólogos, antropólogos, comunicadores, administradores, expertos en mercadeo, en fin, todos los profesionales que eventualmente puedan verse enviueltos en un programa comunitario, deben renunciar a una buena parte de sus tecnicismos, para que el programa pueda salir airoso y cumplir con su objetivo.

En resumen, convencerse de que la receptividad y capacidad de asimilación de los "Pobres" en las ciudades y en los campos es superior a cuanto equivocadamente se dice por falta de un conocimiento objetivo de ellos; saber encontrar y encauzar adecuadamente la insospechable capacidad de voluntariado y de ayuda comunitaria que existe potencialmente entre los "Pobres" rurales y urbanos; tener el valor de adaptarse de tradicionales normas académicas o técnicas y establecer programas cuyos componentes mayoritarios sean más bien el entusiasmo, el sentido común, el deseo de servicio a la comunidad y la apropiada utilización de los recursos existentes.

\section{g) ACCION INMEDIATA hacia una PATERNIDAD MAS RESPONSABLE"}

Se necesita "ACCION". Los "Pobres" no pueden seguir siendo víctimas de una reproducción irracional, mientras que gobernantes y directores de instituciones consumen el tiempo y los recursos discutiendo el problema y argumentando sobre si es o no posible dar un anticonceptivo oral sin prescripción médica en un país que no tiene médicos, cuando saben hace tiempo que de los 50 millones de mujeres que toman píldora en el mundo, más de la mitad lo hacen sin prescripción médica. Hay que actuar ya. Si se cometen algunos errores, en el camino se podrán rectificar. La planificación familiar comunitaria es un permanente experimento, un continuo aprendizaje.

En cualquier parte del mundo, las personas de niveles culturales socio-económicos medianamente altos están controlando voluntariamente su fecundidad hasta acercarla a una proporción apenas de reemplazo.

La tasa de crecimiento vegetativo del mundo, si se toma únicamente como ejemplo el grupo poblacional de nivel medianamente alto, se acerca mucho al cero. En cambio, 
el grupo mayoritario de los "Pobres" sigue en tasas de crecimiento vecinas al tres por ciento $y$ es el que ponderalmente ejerce mayor presión sobre la explosión demográfica tomada a nivel mundial.

No importa repetir que la demografía, la economía mundial, el empleo, la "educación", etc., son problemas que interesan profundamente a los participantes de este simposio o a los estadistas, pero en ningún caso a las humildes familias que viven reducidas a sumicrocosmos de miseria. A ellos hay que hablarles en términos de paternidad responsable, planificación prevención del embarazo indesado, o como se le quiera denominar, y en esa forma lo comprenderán, puesto que es su problema para el cual consciente o incoscientemente buscan solución.
Recordemos, para terminar, que la falta de fondos no es una razón para nó actuar si se los sáve biscar. Habrá siempre los fondos necesarios para programas y proyectos buenos, en tanto que debemos apartarnos de la vieja idea de que habiendo fondos, se generarán por sí mismos los buenos programas. El dinero no hace los programas. Son los programas buenos y bien proyectados los que generan su propia financiación.

\section{SUMARY :}

The present lecture was prepared fo the Tokyo International Simposium, held in Tokyo, Japan, April 4-7, 1977. Its original title was "Action now towards more responsible parenthood worldwide" (Proceeding of the Tokyo International Simposium. Japan Science Society). 